\title{
How a Minimum Time Step and Formation of Initial Causal Structure in Space-Time May Void the Penrose Singularity Theorem, as in Hawkings and Ellis's 1973 Write-Ups
}

\author{
Andrew Walcott Beckwith \\ Physics Department, Chongqing University, Chongqing, China \\ Email: Rwill9955b@gmail.com, abeckwith@uh.edu
}

How to cite this paper: Beckwith, A.W. (2018) How a Minimum Time Step and Formation of Initial Causal Structure in Space-Time May Void the Penrose Singularity Theorem, as in Hawkings and Ellis's 1973 Write-Ups. Journal of High Energy Physics, Gravitation and Cosmology, 4, 485-491.

https://doi.org/10.4236/jhepgc.2018.43026

Received: May 7, 2018

Accepted: July 3, 2018

Published: July 6, 2018

Copyright $\odot 2018$ by author and Scientific Research Publishing Inc. This work is licensed under the Creative Commons Attribution International License (CC BY 4.0).

http://creativecommons.org/licenses/by/4.0/

\section{Abstract}

Using a root finder procedure to obtain $\Delta t$ we use an inflaton value due to use of a scale factor $a \sim a_{\min } t^{\gamma}$ if we furthermore use

$\delta g_{t t} \sim a_{\min }^{2} \cdot \phi_{\text {initial }}$. From use of the inflaton, we initiate a procedure for a minimum scale factor, which would entail the $a_{\min }^{4}<0 \Rightarrow a_{\min } \sim \frac{ \pm \sqrt{\#} \cdot(1+i)}{\sqrt{2}}$, for a sufficiently well placed frequency $\omega$. If the Non Linear Electrodynamics procedure of Camara et al. of General relativity was used, plus the modified Heisenberg Uncertainty principle, of Beckwith, and others, i.e.

$\left.\Delta E \sim\left[\hbar / \Delta t \cdot\left(\delta g_{t t} \sim a_{\min }^{2} \cdot \phi_{\text {initial }}\right)\right]\right|_{\text {Pre-Planckian }} \sim H($ Hubble $)$ we come due to a sufficiently high frequency a case for which $a_{\min } \sim \frac{ \pm \sqrt{\#} \cdot(1+i)}{\sqrt{2}}$ implies a violation of the Penrose singularity theorem, i.e. this is in lieu of $\Lambda_{\text {initial }}=\Lambda_{\text {Today }}$. If this is not true, i.e. that the initial $\Lambda \gg \Lambda_{\text {Today }}$, then we will likely avoid $a_{\min } \sim \frac{ \pm \sqrt{\#} \cdot(1+i)}{\sqrt{2}}$ for reasons brought up in this manuscript.

\section{Keywords}

Inflaton Physics, Causal Structure, Non Linear Electrodynamics

\section{Framing the Initial Inquiry}

Here, the idea would be, to make the following equivalence, namely look at, [1] as well as our own derivation 


$$
\left[\left[\frac{\Lambda_{\mathrm{Max}} r^{4}}{8 \pi G}\right] \cdot(4 / 3) \cdot\left[\frac{2 \pi^{2} g_{*}}{45}\right]^{1 / 3}\right]^{3 / 4} \sim S_{\text {initial }}
$$

We furthermore, make the assumption of a minimum radius of [2] [3]

$$
R_{\text {initial }} \sim \frac{1}{\#} \ell_{N g}<l_{\text {Planck }}
$$

We will initially be assuming that the cosmological constant remains at a constant value, as it is today, and does not change over time (which is the situation given in [4]) as given by Park et al., where the initial value of the cosmological constant could be much higher initially.

This Equation (1) will be put as the minimum value of $r$, where we have in this situation [5] [6]

$$
\text { \# bits } \sim\left[\frac{E}{\hbar} \cdot \frac{l}{c}\right]^{3 / 4} \approx\left[\frac{M c^{2}}{\hbar} \cdot \frac{l}{c}\right]^{3 / 4}
$$

And if $M$ is the total space-time energy mass, for initial condition [5] [6] where

$$
S_{\text {initial }} \sim n_{\text {graviton }} \sim \text { initial graviton count }
$$

$M$ then will be defined by the mass of a massive graviton [7], times, the graviton count, as given in (4) and the modified uncertainty principle, [3] and the Camarra et al. defined Hubble parameter, given in [2]

$$
\begin{aligned}
\Delta E & \left.\sim\left[\hbar / \Delta t \cdot\left(\delta g_{t t} \sim a_{\text {min }}^{2} \cdot \phi_{\text {initial }}\right)\right]\right|_{\text {Pre-Planckian }} \\
& \sim H(\text { Hubble }) \sim \sqrt{\frac{4 \pi G B^{2}}{3 c^{2} \mu_{0}} \cdot\left(1-8 \mu_{0} \omega B^{2}\right)+\frac{\Lambda c^{2}}{3}} \\
& \sim \sqrt{\frac{4 \pi G B^{2}}{3 c^{2} \mu_{0}} \cdot\left(1-8 \mu_{0} \omega B^{2}\right)+\frac{\Lambda_{\text {Today }} c^{2}}{3}}
\end{aligned}
$$

This will lead to

$$
a_{\min } \sim \frac{ \pm \sqrt{\#} \cdot(1+i)}{\sqrt{2}}
$$

whereas if $\Lambda \gg \Lambda_{\text {Today }}$, Equation (6) likely will not hold, and we also state that Equation (6) is a violation of the Penrose singularity theorem as written up in [8], whereas we also have that we are using the Padmanbhan results as given in [9] [10] [11] to the effect that we are employing

$$
\begin{aligned}
& a \sim a_{\text {initial }} t^{\gamma} \\
& \phi=\sqrt{\frac{\gamma}{4 \pi G}} \ln \left[\sqrt{\frac{8 \pi V_{0} G}{\gamma(3 \gamma-1)}} \cdot t\right]
\end{aligned}
$$

While adhering to a potential in line with

$$
V=V_{0} \exp \left[\left\{-\sqrt{\frac{16 \pi G}{\gamma}}\right\} \cdot \phi(t)\right]
$$


We next then go to the results given in [9], which is a publication in JHEPGC by the author, in 2017 .

\section{Examination of the Minimum Time Step, in Pre-Planckian Space-Time as a Root of a Polynomial Equation}

We initiate our work, citing [9] to the effect that we have a polynomial equation for the formation of a root finding procedure for $\Delta t$, namely if

$$
\begin{aligned}
& \Delta t \cdot\left|\left(\sqrt{\frac{8 \pi G V_{0}}{\gamma \cdot(3 \gamma-1)}} \cdot \Delta t-1\right)-\frac{\left(\sqrt{\frac{8 \pi G V_{0}}{\gamma \cdot(3 \gamma-1)}} \cdot \Delta t-1\right)^{2}}{2}+\frac{\left(\sqrt{\frac{8 \pi G V_{0}}{\gamma \cdot(3 \gamma-1)}} \cdot \Delta t-1\right)^{3}}{3}-\cdots\right| \\
& \approx\left(\sqrt{\frac{\gamma}{\pi G}}\right)^{-1} \frac{48 \pi \hbar}{a_{\min }^{2} \cdot \Lambda}
\end{aligned}
$$

From here, we then cited, in [9], using [6] a criteria as to formation of entropy, i.e. If $\Lambda$ is an invariant cosmological "constant" and if Equation (10) holds, we can use the existence of nonzero initial entropy as the formation point of an arrow of time.

$$
\left.S_{\Lambda}\right|_{\text {Arrow-of-time }}=\pi \cdot\left(\frac{\left.R_{c}\right|_{\text {initial }} \sim c \cdot \Delta t}{l_{\text {Planck }}}\right)^{2} \neq 0
$$

This leads to the following, namely in [9] we make our treatment of the existence of causal structure, as given by writing its emergence as contingent upon having

$$
\left(\frac{\left.R_{c}\right|_{\text {initial }} \sim c \cdot \Delta t}{l_{\text {Planck }}}\right) \sim \vartheta(1)
$$

The rest of this article will be contingent upon making the following assumptions. FTR

That we will drop most of the terms in the expansion of Equation (9) and instead of a huge infinite expansion of terms, pick instead using

$$
\Delta t \cdot\left(\sqrt{\frac{8 \pi G V_{0}}{\gamma \cdot(3 \gamma-1)}} \cdot \Delta t-1\right) \approx\left(\sqrt{\frac{\gamma}{\pi G}}\right)^{-1} \frac{48 \pi \hbar}{a_{\min }^{2} \cdot \Lambda}
$$

This is assuming here that the terms in $\left(\sqrt{\frac{8 \pi G V_{0}}{\gamma \cdot(3 \gamma-1)}} \cdot \Delta t-1\right)$ are extremely small, which permits us to come up with a quadratic expression of the term $\Delta t$ which is of course useful as to what we do next, i.e.

If we make use of the Peebles relationship [12] of what would be occurring just before and at the start of expansion of the universe, i.e. the causal structure as given by [9] as, using the Keiffer result of [13] so as to get 


$$
\begin{aligned}
& g_{t t} \sim \delta g_{t t} \approx a_{\text {min }}^{2} \phi_{\text {initial }} \ll 1 \\
& \stackrel{\text { Pre-Planck } \rightarrow \text { Planck }}{\longrightarrow} \delta g_{t t} \approx a_{\text {min }}^{2} \phi_{\text {Planck }} \sim 1 \\
& \left.\Leftrightarrow\left(\frac{\left.R_{c}\right|_{\text {initial }} \sim c \cdot \Delta t}{l_{\text {Planck }}}\right) \sim \vartheta(1)\right|_{\text {Planck }}
\end{aligned}
$$

\section{Consequences, in Terms of the Minimum Scale Factor}

We then use the Peebles result [12] for the strain of space-time at the START of expansion result of

$$
\begin{aligned}
\Delta E & \left.\sim\left[\hbar / \Delta t \cdot\left(\delta g_{t t} \sim a_{\min }^{2} \cdot \phi_{\text {initial }}\right)\right]\right|_{\text {Pre-Planckian }} \\
& \sim H(\text { Hubble }) \sim \sqrt{\frac{4 \pi G B^{2}}{3 c^{2} \mu_{0}} \cdot\left(1-8 \mu_{0} \omega B^{2}\right)+\frac{\Lambda c^{2}}{3}} \\
& \sim \sqrt{\frac{4 \pi G B^{2}}{3 c^{2} \mu_{0}} \cdot\left(1-8 \mu_{0} \omega B^{2}\right)+\frac{\Lambda_{\text {Today }} c^{2}}{3}}
\end{aligned}
$$

The key result is that we have a quadratic expression for the $\Delta t$ term, as indicated by (12) with the result that there is a solvable expression in terms of $\Delta t$, so that then, we can take the square of the terms of Equation (14) with using the expression of Equation (7) above, in order to obtain after using an expansion of Ln $x$, (if $0<x<2$ ) from [14] to get, then, after algebra

$$
\begin{aligned}
& \frac{\gamma}{4 \pi G}\left(\sqrt{\frac{8 \pi G V_{0}}{\gamma \cdot(3 \gamma-1)}} \cdot \Delta t-1\right)^{2} \\
& \sim \frac{\hbar^{2}}{a_{\min }^{4} \cdot(\Delta t)^{2} \cdot\left(\frac{4 \pi G B^{2}}{3 c^{2} \mu_{0}} \cdot\left(1-8 \mu_{0} \omega B^{2}\right)+\frac{\Lambda_{\text {Today }} c^{2}}{3}\right)}
\end{aligned}
$$

This is also reflecting the ideas given in reference [15] which has similar ideas which are similar to our Equation (15) above.

\section{Conclusion, Two Parts}

\subsection{So What If the Denominator of Equation (15) Is Less Than Zero?}

If that happens, due to a very high frequency value for gravitational waves, and a small cosmological constant, we then have

$$
a_{\min }^{4}<0 \Rightarrow a_{\min } \sim \frac{ \pm \sqrt{\#} \cdot(1+i)}{\sqrt{2}}
$$

Note here that when this happens, we have two equally admissible solutions for the scale factor, minimum, and the consequences; if \# is a real number, then we have a contradiction with what is called Theorem 3, Hawking (1967) as cited on page 271 , of [8] we have that

Theorem 3: If $R_{a b} K^{a} K^{b} \geq 0$ for every non space-like Vector $K$

1) The strong casuality condition holds on $(\tilde{M}, g)$, 
2) There is some past-directed unit timelike vector $W$ at a point $p$, and a positive constant $b$, such that if $V$ is the Unit tangent factor to the past directed timelike geodesic through $p$, then on each geodesic the expansion $\theta \equiv V_{; a}^{a}$ of these geodesics becomes less than $-3 c / p$, within a distance $b / c$ from $p$, where $c=-W^{a} V_{a}$, i.e. then there is a past incomplete non space-like geodesic through $p$.

One does not have a curve violating the causality conditions as given as an assertion by Hawkings and Ellis, 1973. i.e. there is, if this occurs at the causal boundary, instead, a bifurcation point at the surface of the causal set, with real and imaginary components, but the incompleteness of the non space geodesic through a point $p$, if it is on the surface of the causal surface, as defined by Equation (13) is not due to a point $p$-. It is well known that certain Kerr black hole models, as in page 465 of Ohanian and Ruffini [14] involve the use of $g_{t t} \sim 0$ for their horizon surfaces and the definition of a plate disc singularity surface but we are instead employing, $\delta g_{t t} \sim a_{\min }^{2} \cdot \phi_{\text {initial }}$.

i.e. precisely because we have avoided using $g_{t t} \sim 0$ as was done in the Kerr black holes, as given in [14] but instead have the $\delta g_{t t} \sim a_{\min }^{2} \cdot \phi_{\text {initial }}$ plus the situation we wish to avoid, that of instead looking at $a_{\min }^{4}<0 \Rightarrow a_{\min } \sim \frac{ \pm \sqrt{\#} \cdot(1+i)}{\sqrt{2}}$, that a causal surface, would be formed on a sphere of space time which would in itself violate the $3^{\text {rd }}$ Penrose theorem.

\subsection{So What Happens If $\Lambda_{\text {initial }} \gg \Lambda_{\text {Today }}$ ?}

The second case to consider would be if we have, instead of today's version of the cosmological constant, a large valued initial cosmological constant, in which then

$$
\begin{aligned}
& \frac{\gamma}{4 \pi G}\left(\sqrt{\frac{8 \pi G V_{0}}{\gamma \cdot(3 \gamma-1)}} \cdot \Delta t-1\right)^{2} \sim \frac{\hbar^{2}}{a_{\text {min }}^{4} \cdot(\Delta t)^{2} \cdot\left(\frac{\Lambda_{\text {initial }} c^{2}}{3}\right)} \\
& \& \Lambda_{\text {initial }} \gg \Lambda_{\text {Today }}
\end{aligned}
$$

We argue that then, there is no reason for assigning a singularity, but it would in line with [4], i.e. assigning an almost infinite value for the initial cosmological constant.

Different variants of the above can be imagined, and of course one should be considering [16] in the reformulation of the Causal structure boundary idea. In addition the points brought up as to [17] to [21] of the nonlinear electrodynamics cosmology should be utilized as a refinement as to the Hubble parameter as outlined in Equation (5) above.

\subsection{Otonion Geometry and Non-Commutativity as a Future Project to Be Combined with Our Present Inquiry?}

We should close with one reference as to the Octonionic geometry program as 
follows. We may be seeing instead of just our roof finder iterations, as outlined above, an exploration into non commutative geometry. This is what I am referring to, and it is from [22].

From [22]

Quote:

i.e.

The change in geometry is occurring when we have first a pre quantum space time state, in which, in commutation relations [23] (Crowell, 2005) in the pre Octonion space time regime no approach to QM commutations is possible as seen by.

$$
\left[x_{j}, p_{j}\right] \neq-\beta \cdot\left(l_{\text {Planck }} / l\right) \cdot \hbar T_{i j k} x_{k} \text { and does not } \rightarrow i \hbar \delta_{i, j}
$$

Equation (18) is such that even if one is in flat Euclidian space, and $i=j$, then

$$
\left[x_{j}, p_{j}\right] \neq i \cdot \hbar
$$

In the situation when we approach quantum "octonion gravity applicable" geometry, Equation (18) becomes

$$
\left[x_{j}, p_{j}\right]=-\beta \cdot\left(l_{\text {Planck }} / l\right) \cdot \hbar T_{i j k} x_{k} \underset{\text { Approaching-flat-space }}{\longrightarrow} i \hbar \delta_{i, j}
$$

End of quote

We assert that the issues as of Equation (18) to Equation (20) if done in higher dimensional analogues, taking into account non commutative initial geometry as outlined in [23] in time, if twinned directly with an analysis of Equation (15) to Equation (17) may in time help us delineate the future of space time research in the early universe.

\section{Acknowledgements}

This work is supported in part by National Nature Science Foundation of China grant No. 11375279.

\section{References}

[1] Kolb, E. and Turner, S. (1994) The Early Universe. Westview Press, Chicago.

[2] Camara, C.S., de Garcia Maia, M.R., Carvalho, J.C. and Lima, J.A.S. (2004) Nonsingular FRW Cosmology and Non Linear Dynamics. Arxiv astro-ph/0402311 Version 1.

[3] Beckwith, A. (2016) Gedanken Experiment for Refining the Unruh Metric Tensor Uncertainty Principle via Schwarzschild Geometry and Planckian Space-Time with Initial Nonzero Entropy and Applying the Riemannian-Penrose Inequality and Initial Kinetic Energy for a Lower Bound to Graviton Mass (Massive Gravity). Journal of High Energy Physics, Gravitation and Cosmology, 2, 106-124. https://doi.org/10.4236/jhepgc.2016.21012

[4] Park, D., Kim, H. and Tamarayan, S. (2002) Nonvanishing Cosmological Constant of Flat Universe in Brane-World Scenario. Physics Letters B, 535, 5-10. https://doi.org/10.1016/S0370-2693(02)01729-X

[5] Ng, Y.J. (2007) Holographic Foam, Dark Energy and Infinite Statistics. Physics Letters $B, 657,10-14$. https://doi.org/10.1016/j.physletb.2007.09.052 
[6] Ng, Y.J. (2008) Spacetime Foam: From Entropy and Holography to Infinite Statistics and Nonlocality. Entropy, 10, 441-461. https://doi.org/10.3390/e10040441

[7] Goldhaber, A. and Nieto, M. (2010) Photon and Graviton Mass Limits. Reviews of Modern Physics, 82, 939-979. https://doi.org/10.1103/RevModPhys.82.939

[8] Hawkings, S.W. and Ellis, G.F.R. (1973) The Large Scale Structure of Space-Time. Cambridge Monographs on Mathematical Physics. Cambridge University Press, New York. https://doi.org/10.1017/CBO9780511524646 https://drive.google.com/file/d/0B7Wa-F5dVHT7cVk2SHJBOHNHeGM/view

[9] Beckwith, A.W. (2017) How a Minimum Time Step Leads to a Causal Structure Used to Form Initial Entropy Production and High Frequency Gravitons, with 7 Subsequent Open Questions. Journal of High Energy Physics, Gravitation and Cosmology, 3, 493-502. https://doi.org/10.4236/jhepgc.2017.33038

[10] Padmanabhan, T. (2005) Understanding Our Universe; Current Status, and Open Issues. In: 100 Years of Relativity, Space-Time, Structure: Einstein and Beyond, World Scientific, P.T.E. LTD, Singapore, 175-204. https://doi.org/10.1142/9789812700988_0007

[11] Padmanabhan, T. (2006) An Invitation to Astrophysics. World Scientific Series in Astronomy and Astrophysics: Volume 8. World Press Scientific, Singapore.

[12] Peebles, P.J.E. (1993) Principles of Physical Cosmology. Princeton University Press, Princeton, New Jersey.

[13] Keifer, C. (2012) Can the Arrow of Time Be Understood from Quantum Cosmology?. In: Mersini-Houghton, L and Vaas, R., Eds., The Arrows of Time, A Debate in Cosmology, Fundamental Theories in Physics, Volume 172, Springer Verlag, Heidelberg, 191-203.

[14] Beyer, W. (1986) CRC Standard Mathematical Tables. 28th Edition, Massachusetts, Boston.

[15] Ohanian, H. and Ruffini, R. (1994) Gravitation and Space-Time. 2nd Edition, WW. Norton, New York.

[16] Dowker, F. (2005) Causal Sets and the Deep Structure of Space-Time. https://arxiv.org/abs/gr-qc/0508109

[17] Rovelli, C. and Vidotto, F. (2015) Covariant Loop Quantum Gravity. Cambridge University Press, Cambridge.

[18] Corda, C. and Mosquera Cuesta, H.J. (2011) INFLATON from $R^{2}$ Gravity: A New Approach Using Nonlinear Electrodynamics. Astroparticle Physics, 34, 587-590.

[19] De Lorenci, V.A., Klippert, R., Novello, M. and Salim, J.M. (2002) Nonlinear Electrodynamics and FRW Cosmology. Physical Review D, 65, Article ID: 063501.

[20] Corda, C. and Mosquera Cuesta, H.J. (2010) Removing Black-Hole Singularities with Nonlinear Electrodynamics. Modern Physics Letters A, 25, 2423-2429.

https://arxiv.org/abs/0905.3298 https://doi.org/10.1142/S0217732310033633

[21] Penrose, R. (1965) Gravitational Collapse and Space-Time Singularities. Physical Review Letters, 14, 57-59. https://doi.org/10.1103/PhysRevLett.14.57

[22] Beckwith, A.W. (2017) Reconsidering "Does the Sum Rule Hold at the Big Bang?" with Pre Planckian HUP, and Division Algebras. Journal of High Energy Physics, Gravitation and Cosmology, 3, 539-557.

[23] Crowell, L. (2005) Quantum Fluctuations of Spacetime. World Press Scientific, Singapore. https://doi.org/10.1142/5952 